\section{Spontanheilung bei Rhinitis allergica?}

\author{
Epidemiologische Studien belegen einen Anstieg allergischer \\ Erkrankungen in den letzten Jahrzehnten. Noch wenig erforscht ist \\ dagegen die Remissionsrate dieser Leiden. In einer Fragebogenstudie \\ wurde ein Zeitraum von acht Jahren abgedeckt.
}

F ür eine Studie verschickten schwedische Wissenschaftler in den Jahren 1992 und 2000 an 4.933 zufällig ausgewählte, in Südschweden lebende Personen per Post einen Fragebogen. Die Befragten mussten darin Angaben zu respiratorischen Symptomen und Erkrankungen der Atemwege machen. 4.280 $(86 \%)$ antworteten auf beide Befragungen. Die Personen waren 1992 zwischen 20 und 59 Jahre alt. Das Vorliegen einer allergischen Rhinitis wurde von den Autoren definiert als Angaben der Patienten, bei Kontakt zu Baum- und Gräserpollen, felltragenden Tieren oder Hausstaub nasale Symptome zu entwickeln. Vom Vorliegen eines Asthmas wurde ausgegangen, wenn die Frage nach einer ärztlichen Diagnose der Erkrankung bejaht wurde.

Die Prävalenz einer allergischen Rhinitis stieg in der Gesamtkohohrte von 12,4\% im Jahr 1992 auf 15,0\% im Jahr 2000. Der Anstieg war auf Neusensibilisierungen gegen Baum- und Grä- serpollen, jedoch nicht gegen Haustiere und Hausstaubmilben zurückzuführen. Die Inzidenz der allergischen Rhinitis betrug im Beobachtungszeitruam im Mittel 4,8\%. Dagegen gaben 23,1\% der Personen, die im Jahr 1992 noch unter allergischer Rhinitis gelitten hatten, bei der zweiten Befragung an, keine Symptomatik mehr zu haben. Sie befanden

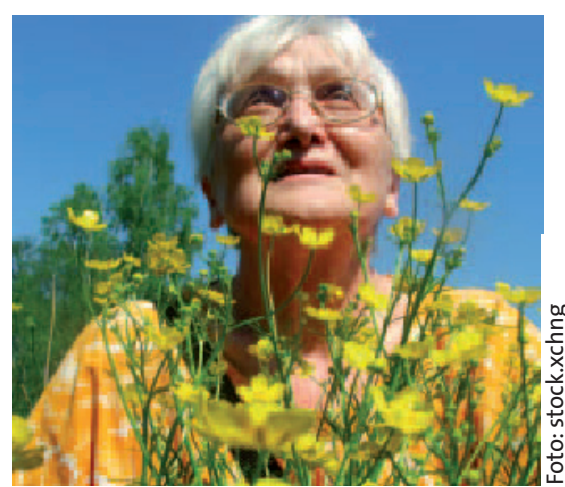

Gute Nachrichten für Heuschnupfenpatienten: Mit den Jahren kann das Leiden ganz von selbst verschwinden. sich damit offensichtlich in Remission. Die höchste Inzidenz einer allergischen Rhinitis wurde bei der jüngsten Gruppe zwischen 20 und 29 Jahren festgestellt, die höchste Remissionsrate in der Gruppe der ältesten Befragten zwischen 50 und 59 Jahren.

Asthmasymptome im letzten Jahr vor der ersten Befragung waren ein Prädiktor für die Entwicklung einer allergischen Rhinitis sowie einer geringeren Chance für eine Remission (Odds ratio [OR] 1,89 bzw. 0,52). Eine positive Familienanamnese für allergische Rhinitis oder Asthma bronchiale erwies sich ebenfalls als Prädiktor (OR 1,99 bzw. 1,62). Eine Assoziation mit der Remissionswahrscheinlichkeit ließ sich bei diesen beiden Faktoren dagegen nicht errechnen. Der Gebrauch von inhalativen Glukokortikoiden erhöhte die Remissionswahrscheinlichkeit ebenfalls nicht.

Fazit: Auch in Südschweden stieg die Prävalenz der allergischen Rhinitis zwischen 1992 und 2000 an. Bei rund 20\% der Befragten, überwiegend bei den über 50-Jährigen, verschwanden die Symptome jedoch in dem Acht-JahresZeitraum wieder.

$b k$

Nihlén $\mathrm{U}$ et al. Incidence and remission of self-reported allergic rhinitis symptoms in adults. Allergy 2006; 61: 1299-304

\title{
Gräserpollentablette im Sicherheitstest
}

\section{Schnell lösliche Tabletten sollen bei der sublingualen Immuntherapie (SLIT) eine Alternative zu den gewohnten Tropfen bilden. Eine Phase-I- Studie prüfte jetzt die Sicherheit einer neuen Gräserpollentablette.}

$\mathrm{n}$ einer dänisch-französischen Studie

sollte die Verträglichkeit einer neu entwickelten Gräserallergentablette untersucht werden. Dazu wurden 30 Patienten mit Gräserpollenallergie auf vier Gruppen verteilt, die die Tablette an zehn aufeinanderfolgenden Tagen in jeweils anderer Dosierung einnahmen. Gruppe eins erhielt die Tabletten-Immuntherapie in ansteigenden Dosierungen, die jeden zweiten Tag erhöht wurden (100, 200, 300, 400 und 500 IR). Gruppe zwei erhielt die Tabletten-SLIT in täglich an- steigenden Dosierungen (100, 200, 300, 400 und 500 IR) mit anschließender Erhaltungsdosis von 500 IR über fünf weitere Tage. Gruppe drei wurde konstant über alle zehn Tage mit einer Gräserallergendosis von 300 IR und Gruppe vier konstant mit 500 IR behandelt.

In keiner der Gruppen traten schwere systemische Nebenwirkungen auf. In der konstant mit der höchsten Allergendosis behandelten Gruppe vier wurden aber schwere lokale unerwünschte Ereignisse wie Schwellungen im Rachen beobachtet. In den Gruppen eins bis drei traten nur milde bis mäßig starke, transiente lokale Reaktionen auf, wie z. B. oraler Pruritus, Reizung des Rachens und Schwellung der Zunge.

Fazit: Die getestete Allergentablette führte zu keinen schweren systemischen Nebenwirkungen. Eine von Beginn an hohe Dosierung mit 500 IR scheint ein ungünstigeres Sicherheitsprofil zu besitzen als eine rasche Aufdosierung auf eine Erhaltungsdosis von 500 IR. Diese wurde gut vertragen mit überwiegend leichten lokalen Nebenwirkungen.

$f k$

Larsen TH et al. Safety and tolerability of grass pollen tablets in sublingual immunotherapy - a phase-1 study. Allergy 2006; 61: 1173-6 